\title{
Long Noncoding RNA AATBC Promotes the Proliferation and Migration of Prostate Cancer Cell Through miR-1245b-5p/CASK Axis
}

\author{
Wenyuan Zhang' \\ Qionghong $\mathrm{Liu}^{2}$ \\ Jun Zhao' \\ Tiejun Wang ${ }^{2}$ \\ Jinshan Wang ${ }^{2}$ \\ 'Department of Urology, East Hospital, \\ Ji'an Hospital, Jiangxi, 343000, People's \\ Republic of China; ${ }^{2}$ Department of \\ Urology, East Hospital, Tongji University \\ School of Medicine, Shanghai, 200I23, \\ People's Republic of China
}

Correspondence: Jinshan Wang

Department of Urology, East Hospital, Tongji University School of Medicine, Shanghai, 200123, People's Republic of China

Email wangjinshandoctor@aliyun.com
Introduction: Long noncoding RNAs (lncRANs) as suppressive or oncogenic genes have been substantiated in prostate cancer $(\mathrm{PCa})$. In the current study, the role and molecular mechanism of lncRNA AATBC in the progression of PCa was evaluated.

Methods: LncRNA AATBC and miR-1245b-5p expression were evaluated using RT-qPCR. CCK-8, colony-formation, apoptosis and transwell assay were used to analyze the in vitro role. The xenograft model was used to explore the in vivo role. Bioinformatics analysis and a dual luciferase assay, RIP and RNA pull down were used to confirm the interaction between lncRNA AATBC and 1245b-5p, as well as 1245b-5p and CASK.

Results: Firstly, we certified that the expression of AATBC was augmented in PCa, and knockdown of AATBC could significantly inhibit the growth of PCa in vitro and in vivo. Mechanistically, our results manifested that AATBC could directly bind to miR-1245b-5p. In addition, miR-1245b-5p played cancer-suppressive role in PCa cells. Moreover, CASK was attested as the target of miR-1245b-5p, and CASK was demonstrated to exert as oncogene in the progression of PCa. Finally, rescue assays illustrated that miR-1245b-5p downregulation or CASK restoration could greatly resist the restrained effects of AATBC knockdown on PCa progression.

Conclusion: AATBC could accelerate the progression of PCa through regulating miR1245b-5p/CASK axis, which provided a potential therapeutic target for PCa treatment.

Keywords: lncRNA, prostate cancer, AATBC, miR-1245b-5p, CASK

\section{Introduction}

Prostate cancer $(\mathrm{PCa})$ is one of the most common and fatal malignancies in men worldwide, and the incidence increases significantly with age. ${ }^{1}$ Patients with early PCa can achieve a good prognosis after systemic androgen deprivation therapy or hormonal therapy, surgical resection. ${ }^{2}$ However, patients with advanced PCa often have a significant decrease in survival time and quality of life because of metastasis, recurrence and drug resistance. ${ }^{2,3}$ It is therefore essential to carry out comprehensive exploration and mine novel molecular mechanisms in order to find new targets for the treatment of PCa.

Voluminous studies have spotted that non-coding RNAs impersonate a sizeable role in tumor formation and progression, including small noncoding RNAs and long noncoding RNAs (lncRANs), both of which are a class of non-coding RNAs without protein-coding ability. ${ }^{4,5}$ Among them, lncRNAs are non-coding RNAs greater than 200 nucleotides in length, and plentiful researches have reported that 
IncRNAs can affect growth, angiogenesis, cycle and apoptosis, autophagy, migration and invasion, and metabolism in different tumors. ${ }^{6-9}$ As an example, lncRNA PVT1 activated STAT3/VEGFA signal pathway to induce angiogenesis ${ }^{10}$ and enhances the progression of gastric cancer by the downregulation of p15 and p16. ${ }^{11}$ LncRNA FOXD1-AS1 boosts glycolysis of nasopharyngeal carcinoma by the regulation of related glycolytic genes ${ }^{12}$ and regulates the growth and metastasis of glioma via miR339/ 342-eIF5a axis. ${ }^{13}$ Likewise, the functions of lncRNAs in PCa have also been largely reported. For instance, lncRNA LEF1-AS1 upgrades androgen-independent PCa progression via the upregulation of FZD2 and CD44 ${ }^{14}$ and lncRNA HOTAIR advances the invasion and metastasis of PCa by activating MAPK pathway. ${ }^{15}$

LncRNA AATBC (apoptosis-associated transcript in bladder cancer, LOC284837) firstly was reported in bladder cancer, and knockdown of lncRNA AATBC inhibited proliferation and induced apoptosis in bladder cancer. ${ }^{16}$ In addition, Tang et al found lncRNA AATBC promoted growth and metastasis of nasopharyngeal carcinoma. ${ }^{17}$ However, the character of AATBC in PCa is still undefined. Consequently, we probed into the action and potential molecular mechanism of AATBC in PCa.

\section{Materials and Methods}

\section{Clinical Tissue Specimens}

The tumor tissues and neighboring normal prostate tissues of 86 patients with PCa were gathered from January 2016 to December 2018 at East Hospital, Tongji University School of Medicine, Shanghai. All samples were stored at $-80^{\circ} \mathrm{C}$ and used to the following experiments. All subjects did not experience any treatments such as chemotherapy, targeted therapy, or immunotherapy et al prior to obtain tissue samples. The research was approbated by the ethics committee of East Hospital, Tongji University School of Medicine, Shanghai, and all patients who participated in the research inscribed informed consent, and the research was conducted in accordance with the Declaration of Helsinki.

\section{Cell Lines and Culture}

The normal human prostate epithelial cell line (RWPE-1) was purchased from the American type culture collection (ATCC) (Maryland, USA). The human PCa cell lines (LNCaP, DU145, 22RV1, VCaP, PC3) were acquired from Cell Bank of Chinese Academy of Sciences
(Shanghai, China). PC3 and VCaP cells were grown in F12-K medium (life science, USA) and DMEM medium (Gibco, USA), respectively. LNCaP, DU145 and 22RV1 were cultured in RPMI 1640 (life science, USA). All medium were added with $10 \%$ FBS (Invitrogen, Waltham, MA, US) and penicillin/streptomycin (100 U/ $\mathrm{mL}$, Sigma). Keratinocyte Serum Free Medium (Invitrogen, Waltham, MA, US) was provided to culture RWPE-1 cells. All the cells were placed at $37^{\circ} \mathrm{C}$ with a humidified $5 \% \mathrm{CO}_{2}$ incubator.

\section{Oligonucleotides, Plasmids, and Cell Transfection}

Specific shRNAs against AATBC (sh-AATBC), CASK (sh-CASK) and the corresponding negative control (shNC) were purchased by Invitrogen (Shanghai, China). The mimics/inhibitor of miR-1245b-5p and the corresponding negative control were purchased from Shanghai Genechem (Shanghai, China). For CASK overexpression, pcDNA3.1-CASK vectors (oe-CASK) were constructed by Genepharma (Shanghai, China). The siRNAs or vectors were transfected to $\mathrm{PC} 3$ and $\mathrm{LNCaP}$ cells by Lipofectamine 3000 (Thermo Fisher Scientific, USA).

\section{RNA Extraction and qRT-PCR}

The nuclear and cytoplasmic RNAs of cells were extracted by using the nuclear and cytoplasmic extraction reagents (NEPER, Thermo Scientific). Total RNAs were separated by TRIzol reagent (Sigma, USA). Then, the complementary DNA was synthesised by the OneStep PrimeScript miRNA cDNA Synthesis Kit (Takara, Japan). Subsequently, qRT-PCR was performed using QuantiFast SYBR GREEN PCR Kit (Qiagen) on LightCycler96 system (Roche). GAPDH or U6 was used as an endogenous calibrator control of mRNA expression and cytoplasm control as well as the internal reference for miRNA expression and nuclear control, respectively. All primers were synthesized by GenScript (Nanjing, China). The primer sequences were as follows: AATBC, forward: 5'-G GACTGACATTGGTTACGTGG-3', reverse 3'-TCGGCCT GAAGATGCCAATAGG-5'; CASK, forward: 5'-C AATGCTTATGGCGTAATTGGCC-3', reverse, 5'GCTTACAGCCGGAAGGTACGAC-3'; miR-1245b-5p, forward: 5'-TCGTTAGGCCTAGCTGCATTAAC-3'; reverse, 5'-CCGTAGTTAGGCATCGTGTTAGGCTTTTGCCAG-3'; GAPDH, forward: 5'-GTCATCCCTGAGCTAGACGG-3', reverse, 5'-GGGTCTTACTCCTTGGAGGC-3'; U6, forward: 
5'-CTCGCTTCGGCAGCACATATACT-3', reverse, 5'ACGCTTCACGAATTT-GCGTGTC-3'.

\section{CCK-8 Assay}

Briefly, the transfected cells $\left(2 \times 10^{3}\right.$ cells/well $)$ were seeded into 96-well plates. CCK-8 solution of $10 \mu \mathrm{L}$ (CCK-8, Dojindo; Kumamoto, Japan) was added to the well after growing for $24 \mathrm{~h}, 48 \mathrm{~h}, 72 \mathrm{~h}, 96 \mathrm{~h}$, and then the OD450 value was detected by microplate reader (VICTOR, Nivo).

\section{Colony Forming Assay}

Transfected cells (at the density of 500 cells per well) were cultured in 6-well plates. After 2 weeks, 5\% PFA was used to fix the cells and $1 \%$ crystal violet solution was used to stain the cells and ultimately the visible colonies number were calculated.

\section{Flow Cytometry Analysis for Cell Apoptosis}

Cell apoptosis was measured through the Annexin V-FITC/PI Apoptosis Detection Kit (TransGen, Perking, China). Briefly, cells were washed and collected $48 \mathrm{~h}$ after transfection. Next, Annexin V-FITC/PI was used to double stain the cells. Subsequently, flow cytometry (FACScan; BD Biosciences, Franklin Lakes, NJ, USA) was manipulated to monitor the apoptosis.

\section{Migration and Invasion Assay}

The transwell assay was used to detect the ability of cell migration and invasion. For invasion assay, the upper chamber needed to be precoated with matrigel, while it was not required for migration assay. Briefly, transfected cells in serum-free medium were seeded onto the inserts. After $24 \mathrm{~h}$ of induction in medium containing $20 \% \mathrm{FBS}$, the cells at the bottom of the inserts were fixed with 5\% PFA. Staining was subsequently performed with $0.3 \%$ crystal violet. Finally, photographs were taken under a microscope. Five fields were randomly selected and the number of migrated or invaded cells was manually calculated.

\section{Tumor Formation Experiments}

The animal experiments were approved by the ethics committee of East Hospital, Tongji University School of Medicine, Shanghai. We performed the subcutaneous injection of PC3 cells transfected with sh-AATBC or sh-
$\mathrm{NC}$ at the armpits of six-week Balb/c nude female mice that were purchased by Shanghai Model Organisms Center, Inc. (Shanghai, China) ( $\mathrm{n}=6$ for each group). The tumor volumes were scaled every 3 day, and the tumors were harvested and photographed. All experiments in vivo were conducted in compliance with the guidelines for the Care and Use of Laboratory Animals of Tongji University Experimental Animal Center.

\section{Fluorescence in situ Hybridization (FISH) Assay}

PC3 and LNCaP cells were fixed, permeabilized and then hybridized with AATBC-specific FISH probe that were purchased by Invitrogen. Nucleus was dyed with DAPI (Sigma-Aldrich). Fluorescence images were captured using a confocal laser microscope (Olympus).

\section{Dual-Luciferase Reporter Assay}

The regions of AATBC and CASK-3'-UTR containing the wild-type (Wt) or mutant (Mut) miR-1245b-5p binding site were chemically generated by Genechem (Shanghai, China). And then they were cloned into the pmirGLO luciferase reporter gene (Promega, Madison, WI, USA), and named as AATBC-Wt/Mut and CASK-Wt/Mut. Cells were co-transfected with the indicated plasmids and miR1245b-5p mimics using Lipofectamine 3000 reagent (Invitrogen). Luciferase activity was appraised with a Dual-Luciferase Reporter System (Promega, Madison, WI, USA).

\section{RNA Pull Down}

Biotinylated probes with wild-type or mutated miR-1245b5p (Bio-miR-1245b-5p-WT/Mut) or control probe (Bio-NC) were synthesized (Invitrogen, Shanghai). The biotin-labeled RNAs were incubated with streptavidin-coated magnetic beads and the cell lysates (Promega, Madison, WI, USA) at $4^{\circ} \mathrm{C}$ for $4 \mathrm{~h}$. The bound RNAs were purified through the washing of beads and extracted with TRIzol reagent. Finally, the RNAs were detected through qRT-PCR analysis.

\section{RNA Immunoprecipitation (RIP) Assay} Imprint ${ }^{\circledR}$ RNA Immunoprecipitation Kit (Sigma-Aldrich) was applied to conduct the RIP assay. Cells after transfection were lysed with RIP buffer. Subsequently, the protein A magnetic beads conjugated with a human anti-AGO2 antibody or control IgG were mixed with the cell lysate. 
The RNA was extracted by TRIzol reagent and the qRTPCR was performed for the quantified analysis.

\section{Western Blot Analysis}

Briefly, total protein was acquired using RIPA lysis buffer (Sigma, USA) and then the protein was quantified with BCA Protein Assay Kit (Sigma-Aldrich). Subsequently, equivalent amounts of protein were separated by SDSPAGE, transferred to PVDF membrane (Millipore, USA) under $300 \mathrm{~mA}$ for 1.5 hours, then blocked with 5\% BSA, and incubated with primary antibodies purchased from Cell Signaling Technology. The primary antibodies were as follows: CASK (Rabbit mAb\#8968; 1:1000) and GAPDH (Rabbit mAb\#5174; 1:1000 dilution). After the incubation for overnight at $4^{\circ} \mathrm{C}$, the membrane was incubated with secondary antibody (HRP-linked antibody\#7074; 1:3000 dilution; Cell Signaling Technology) at room temperature for $2 \mathrm{~h}$. Eventually, the protein bands were observed with the chemiluminescence system (GE Healthcare, Chicago, USA).

\section{Statistical Analysis}

All experiments results were obtained from triplicates and data were presented as mean \pm SD. GraphPad Prism 7 (La Jolla, CA, USA) was used for data analysis and plotting. Student's $t$-test was used for difference analysis between two groups; one-way analysis of variance (ANOVA) was used for difference analysis between multiple groups. Pearson's correlation analysis was used to weigh the relationship between AATBC and miR-1245b-5p expression in prostate cancer tissues. $\mathrm{P}$ value was less than 0.05 , and the results were statistically significant.

\section{Results}

\section{LncRNA AATBC Expression Was} Notably Up-regulated and Its Knockdown Prohibited the Growth in PCa

Through qRT-PCR analysis, we first demonstrated that the expression of AATBC was considerably accelerated in PCa patients $(n=86)$ than adjacent normal tissues $(n=86)$ (Figure 1A). Identically, the expression of AATBC was also greatly overexpressed in five PCa cell lines (LNCaP, DU145, 22RV1, VCaP, PC3) compared to normal human prostate epithelial cell line (RWPE-1) (Figure 1B). To further appraise the role of AATBC in $\mathrm{PCa}$, we designated two cell lines (PC3 and $\mathrm{LNCaP}$ ) with relatively high expression of AATBC to carry out loss-of-function assay.
Then, the silencing efficiency of AATBC was validated (Figure 1C). CCK-8 assay signified that AATBC deficiency could dramatically prevent the $\mathrm{PCa}$ cell proliferation (Figure 1D and E). Likewise, colony formation assay denoted that the independent growth competence of PC3 and $\mathrm{LNCaP}$ cells was restrained through silencing lncRNA AATBC (Figure 1F). In addition, we also observed that the apoptotic cell proportion in the AATBC silencing PC3 and LNCaP cells were enormously expanded (Figure 1G). Furthermore, transwell assays revealed that the migratory and invasive potency of $\mathrm{PC} 3$ and $\mathrm{LNCaP}$ cells were markedly arrested by AATBC knockdown (Figure 1H and I). Last, xenograft experiments exhibited that AATBC knockdown could remarkably shrink the tumor volumes (Figure 1J). Collectively, AATBC was overexpressed and its knockdown arrested cell growth in PCa cells in vitro and in vivo, which sufficiently manifested an oncogenic role in the progression of $\mathrm{PCa}$ cells.

\section{MiR-1245b-5p Was a Target of AATBC and Suppressed the Development of PCa}

We firstly analyzed the location of AATBC through subcellular fractionation and FISH analysis. As illustrated in Figure $2 \mathrm{~A}$ and $\mathrm{B}, \mathrm{AATBC}$ was mostly located in the cytoplasm of PC3 and LNCaP cells. Hence, we presumed AATBC might function as a competing endogenous RNA (ceRNA) through sponging miRNA to affect the expression of downstream target genes. Whereafter, starBase 3.0 database (http://starbase.sysu.edu.cn/) was applied to predict the miRNAs that may be sponged by AATBC, and we found that 4 miRNAs (miR-3142, miR-3194-5p, miR371a-3p, miR-1245b-5p) possessed the potential binding ability with AATBC. Then, the expression of 4 miRNAs in $\mathrm{PCa}$ cell lines and the normal human prostate epithelial cell line (RWPE-1) was evaluated through qRT-PCR, which showed that miR-1245b-5p had the lowest expression among 4 miRNAs in all PCa cell lines (Figure 2C). In addition, Pearson correlation analysis validated that the expression of miR-1245b-5p and AATBC exhibited the reversed relationship in $\mathrm{PCa}$ tissues (Figure 2D). Therefore, miR-1245b-5p was selected as the target of AATBC. The binding site between the miR-1245b-5p and AATBC was exhibited in Figure 2E. To further confirm the interaction between the miR1245b-5p and AATBC, we first performed the biotin-labeled RNA pull down assay, and the data showed that the expression of AATBC was dramatically upregulated in the PC3 and 

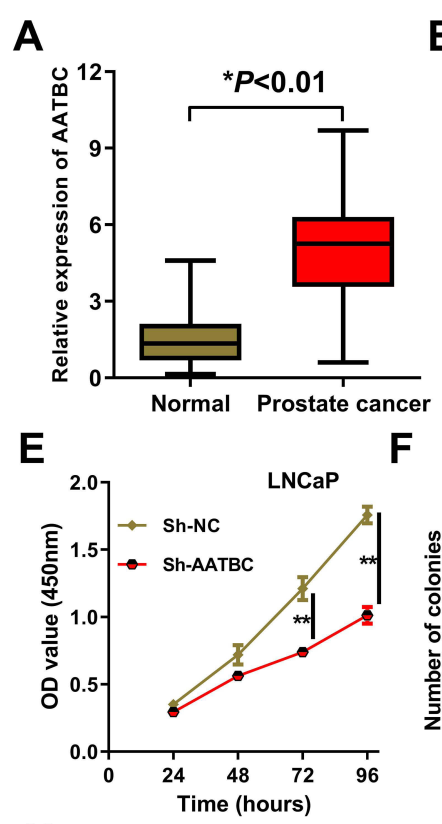

$F$
B

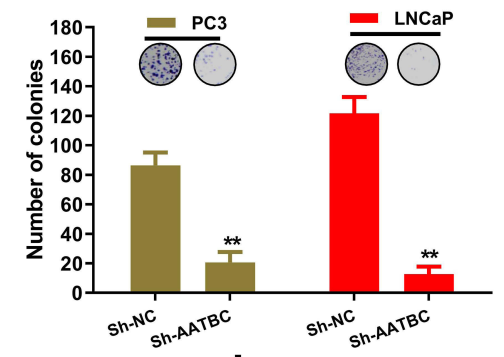

I
C

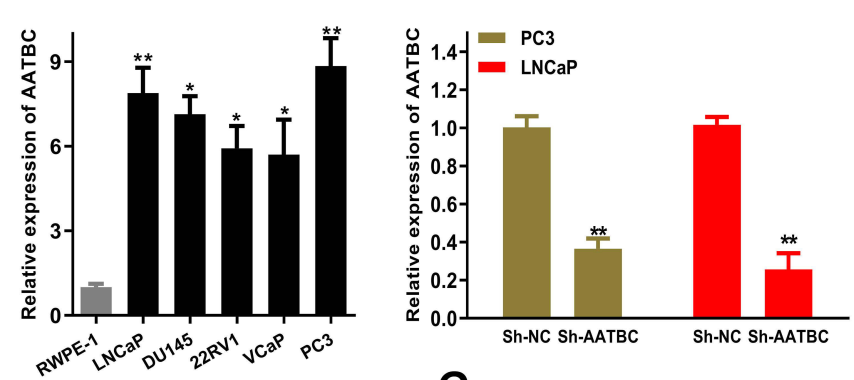

G
D

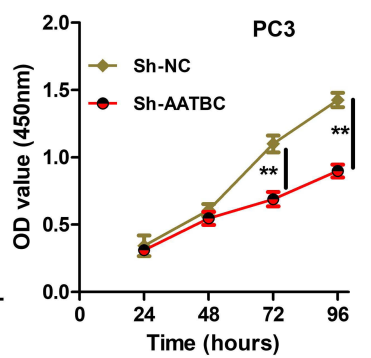

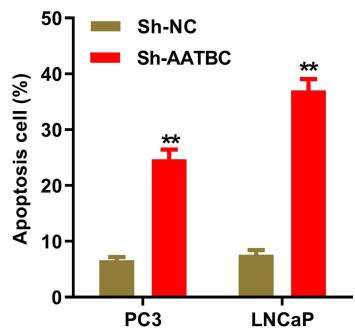

J
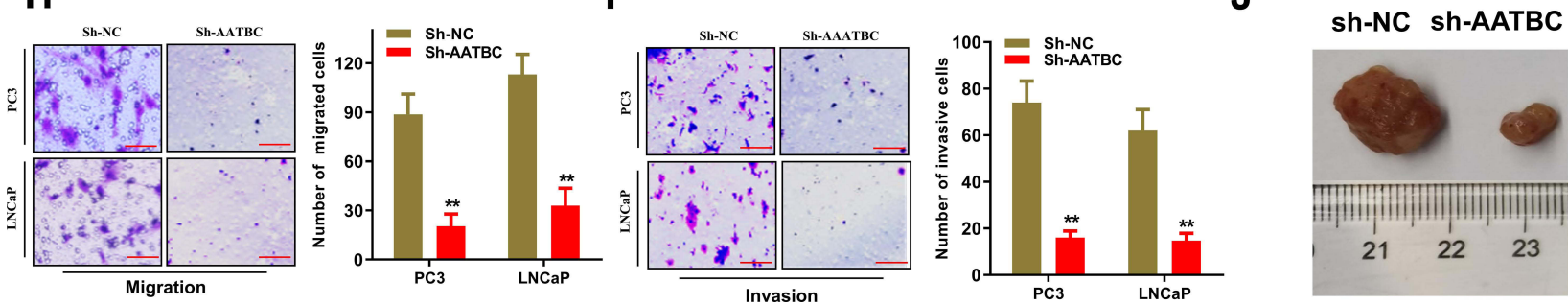

Figure I LncRNA AATBC was significantly up-regulated and its knock-down dramatically inhibited the growth of PCa in vitro and in vivo. (A) Expression of AATBC was detected by qRT-PCR in PCa tissues $(n=86)$ and adjacent normal tissues $(n=86)$. (B) Expression of AATBC was significantly upregulated in PCa cells by qRT-PCR analysis. (C) The validity of AATBC silence was confirmed by qRT-PCR. (D-F) The proliferation and colony formation abilities in PC 3 and LNCAP cells of AATBC knockdown were obviously repressive. (G) The apoptosis in AATBC-silenced PC3 and LNCAP cells were disclosed through Annexin V-FITC/PI apoptosis detection. (H-I) Upon downregulation of AATBC expression, the migration and invasion abilities of PC3 and LNCAP cells were measured by transwell assay (scale bars: $100 \mu \mathrm{m}$ ). (J) Knocking down of AATBC significantly shrunk the volume of xenografted tumor in vivo compared to the negative control with transfected with sh-NC. $* \mathrm{P}<0.05$; $* * \mathrm{P}<0.0$ I.

LNCaP cells transfected with the wild-type biotin-labeled miR-1245b-5p, whereas almost no change in the corresponding mutated biotin-labeled miR-1245b-5p group (Figure 2F). Similarly, luciferase reporter assay ascertained that the overexpression of miR-1245b-5p could significantly suppress the activity of AATBC-WT reporter, nevertheless, no evident variation was observed in AATBC-Mut reporter (Figure 2G). Then, RIP assay revealed that AATBC and miR-1245b-5p was aggregated on the Ago2 precipitates (Figure $2 \mathrm{H}$ ), which further confirmed their interactions in $\mathrm{PCa}$ cells. The overexpression of miR-1245b-5p was validated by qRT-PCR (Figure 2I). As shown in Figure 2J-O, the overexpression of miR$1245 b-5 p$ obviously inhibited the proliferation and colony formation, promoted the apoptosis, and suppressed the migration and invasion in PC3 and LNCaP cells, which certified that miR-1245b-5p exerted a suppressive role in the progression of $\mathrm{PCa}$ cell. In short, miR-1245b-5p was sponged by AATBC and restrained the development of PCa.

\section{CASK Was Targeted by miR-1245b-5p and Promoted the Progression of $\mathrm{PCa}$}

We further explored the putative targets of miR-1245b-5p through bioinformatics analysis from four databases (Starbase, miRDB, Targetscan and miRWalk). Venn diagram indicated 5 candidate mRNAs (SOS2, DHX40, KLHL29, SIK1, CASK) could be targeted by miR$1245 b-5 p$ in Figure 3A. Then, qRT-PCR assay further confirmed that either miR-1245b-5p overexpression or AATBC silencing was able to down-regulate CASK expression rather than other candidate genes in a series of prostate cancer cells (Figure 3B and C). Also, CASK was outstandingly overexpressed in mRNA and protein levels in PCa cells compared with the normal prostate epithelial cell line (RWPE-1) (Figure 3D and E). RIP 

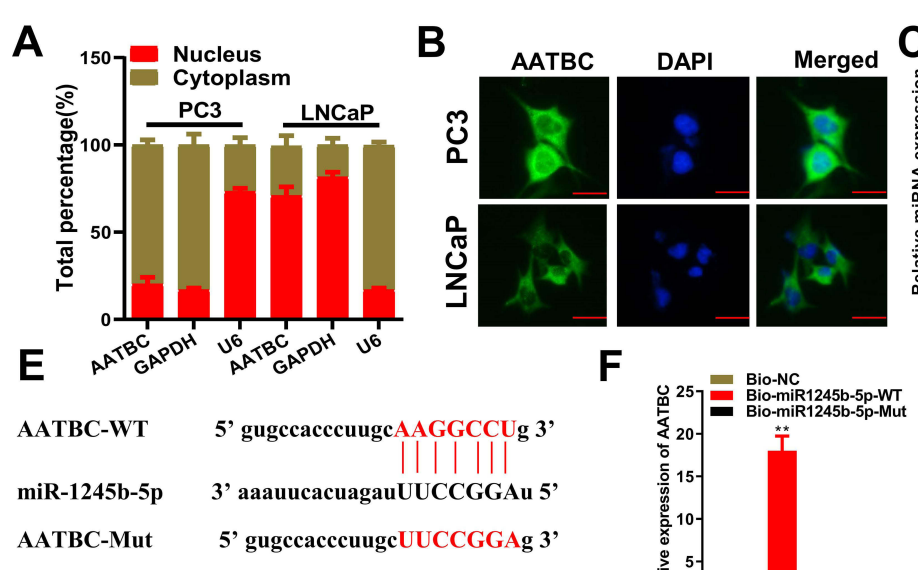

$\mathbf{F}$
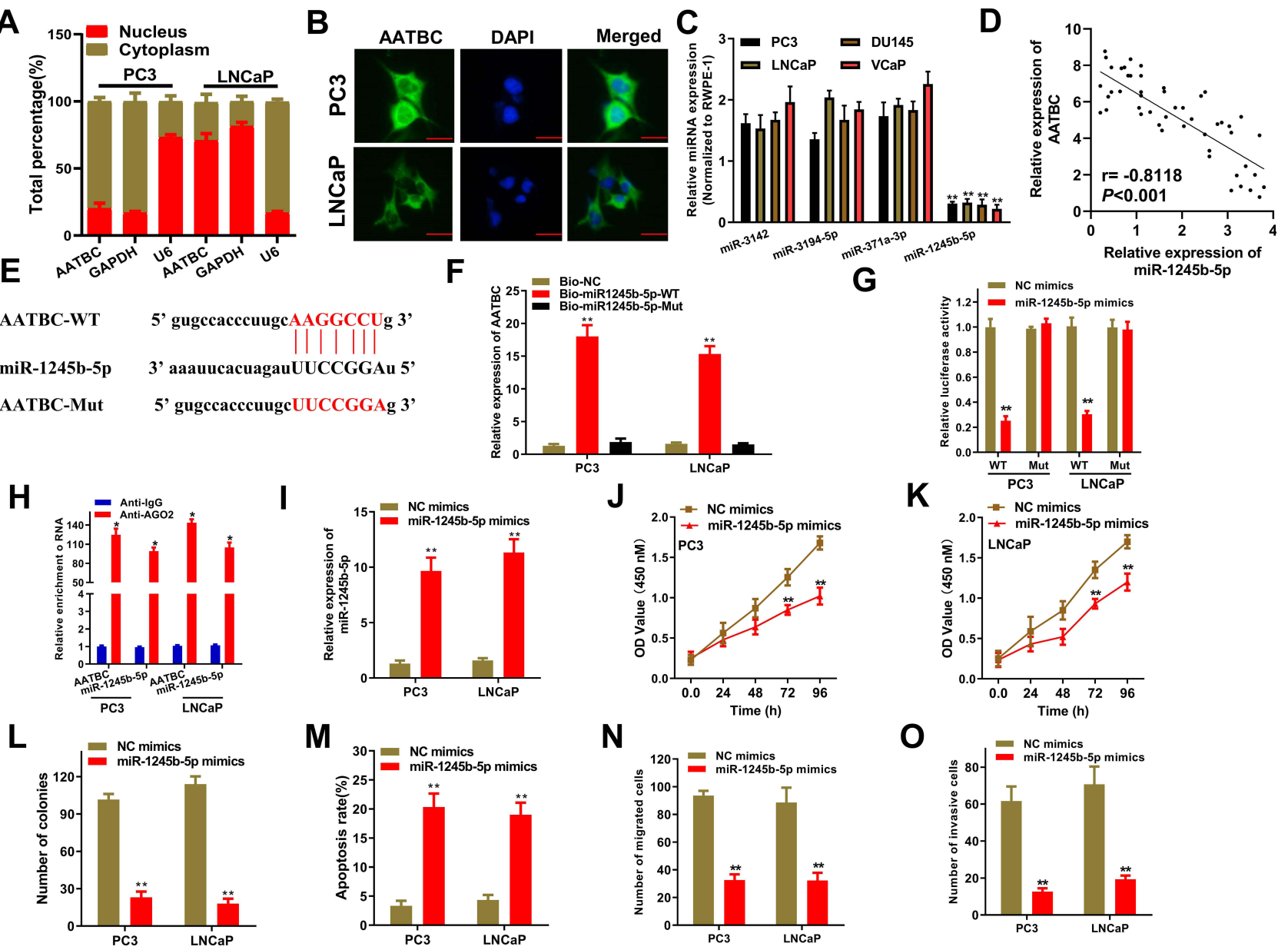

G

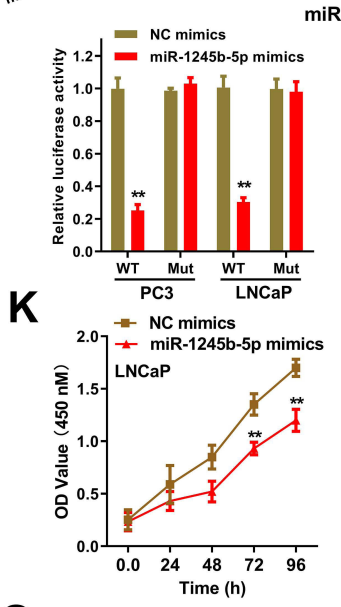

Figure 2 miR-1245b-5p was sponged by AATBC and restrained the progression of PCa. (A and B) Subcellular location of AATBC was determined by nucleus-cytoplasm fractionation and FISH. Scale bars, $20 \mu \mathrm{m}$. (C) Starbase database predicted the four miRNAs that had the binding sites to AATBC, and their expression were determined in the PCa cells by qRT-PCR. (D) Pearson correlation analysis showed the passive relationship between miR-1245b-5p and AATBC expression in PCa tissues from our cohort. (E) Predicted binding sites between miR-1245b-5p and AATBC 3'-UTR. (F-H) Biotin-labeled pull-down assay, luciferase assay and RIP assay were used to affirm the interaction between the miR-1245b-5p and AATBC, respectively. (I) The competence of miR-I245b-5p overexpression was validated by qRT-PCR. (J-L) The proliferation and colony formation abilities in miR-1245b-5p overexpressed PC3 and LNCAP cell were significantly augmented by CCK8 and colony formation assay analysis. (M) miR1245b-5p overexpression significantly promoted the apoptosis of PC3 and LNCAP cells through Annexin V-FITC/PI apoptosis detection. ( $\mathbf{N}$ and $\mathbf{O}$ ) miR-1245b-5p overexpression significantly inhibited the migrated and invasive abilities of PC3 and LNCAP cells by transwell assay, respectively. $* \mathrm{P}<0.05 ; * * \mathrm{P}<0.0 \mathrm{I}$.

assay also validated that CASK, miR-1245b-5p, and AATBC were specially enriched in the Ago2 precipitates (Figure 3F). These results replied that CASK could be the only target of miR-1245b-5p. The conjunctive sequences for miR-1245b-5p and CASK were displayed in Figure 3G. According to this prediction, luciferase reporter assay made clear that luciferase activity of CASK-WT reporter harboring the wild-type miR-1245b-5p-binding site was markedly depressed in $\mathrm{PC} 3$ and PNCaP cells with the miR-1245b-5p overexpression, while luciferase activity of CASK-Mut reporter failed to present obvious alteration (Figure $3 \mathrm{H}$ ). Besides, the biotin-labeled pulldown assay exhibited a potent aggregation of CASK in the biotinylated miR-1245b-5p-WT pulled down pellet by qRT-PCR rather than biotinylated miR-1245b-5p-Mut
(Figure 3I). In addition, the transfected potency of miR1245b-5p inhibitor was authenticated (Figure 3J). Curiously, the lowered CASK mRNA and protein expression provoked by AATBC knockdown could be reestablished through the downregulation of miR-1245b-5p (Figure $3 \mathrm{~K}$ and $\mathrm{L}$ ). After confirming CASK knockdown efficiency in Figure 3M, the impacts of CASK depletion on progression of $\mathrm{PCa}$ were exposed by functional assay. As shown in Figure 3N, CASK knockdown dramatically inhibited the colony formation of PCa cells. And CASK depletion significantly augmented the apoptosis of $\mathrm{PCa}$ cells (Figure 3O). Meanwhile, CASK deficiency markedly discouraged the migration and invasion of $\mathrm{PCa}$ cells (Figure 3P and Q). Taken together, CASK was targeted by miR-1245b-5p and played an oncogenic role in PCa. 

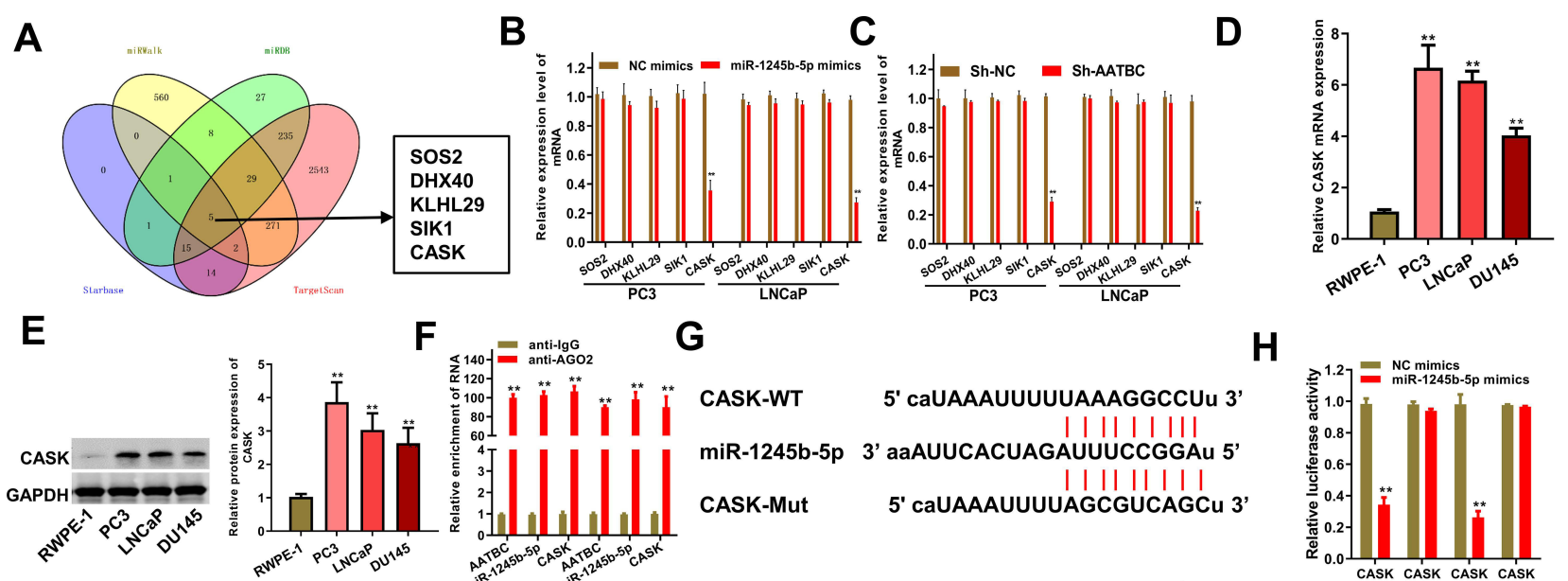

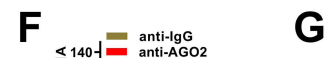
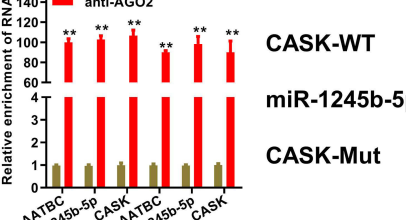

I | || || ||

CASK-Mut

5' caUAAAUUUUAGCGUCAGCu 3'

H
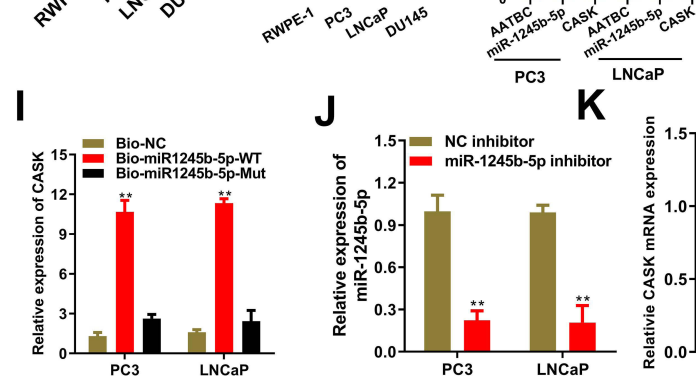

$K^{\mathrm{LNC} C P}$
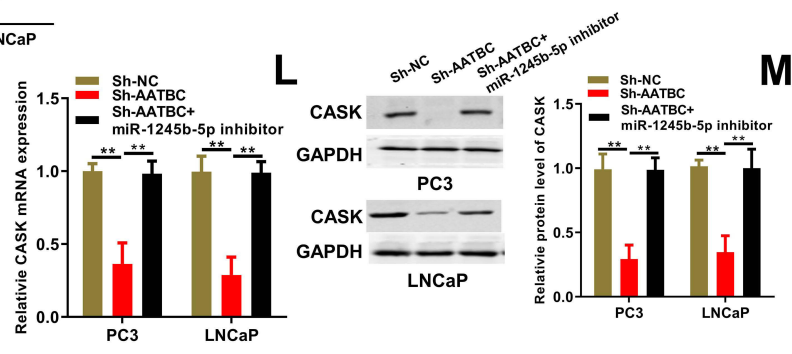

M

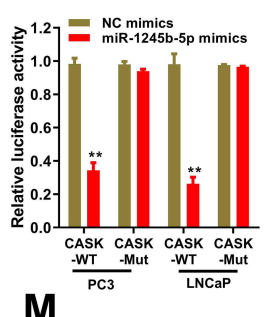

\section{$\mathbf{N}$}

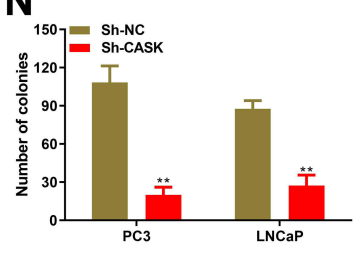

0

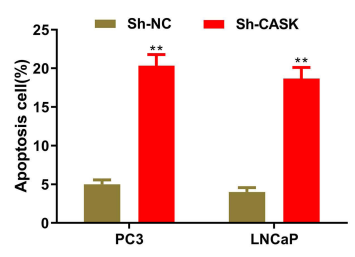

$\mathbf{P}$
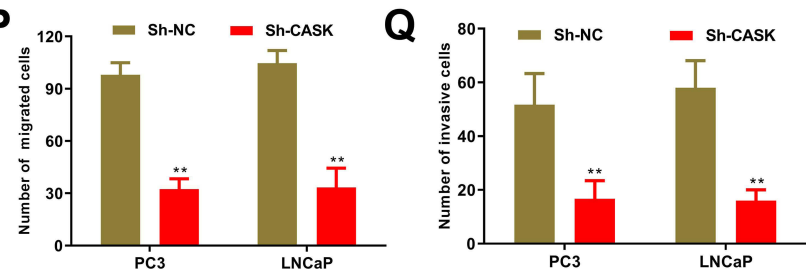

Figure 3 CASK was one downstream target of miR-1245b-5p and boosted the progression of PCa. (A) Venn diagram exhibited the predicted target genes of miR-1245b-5p by the crosstalk of four databases (starbase, miRDB, targetscan and miRWalk), and 5 common genes were selected as the candidate genes. (B and C) qRT-PCR assays confirmed that the expression of CASK could be altered by the overexpression of miR-1245b-5p and silence of AATBC in PC3 and LNCAP cells. (D and E) The mRNA and protein expression of CASK was quantified in PCa cells and normal cells by qRT-PCR and Western blot, respectively. (F) RIP assay justified that AATBC, miR-1245b-5p and CASK could be coexisted in Ago2 pallets. (G) Bioinformatics database predicted the binding site between miR-1245b-5p and CASK. (H and I) Luciferase assay and RNA pull down assay elucidated the interaction between the miR-1245b-5p and CASK above the binding site. (J) The knockdown efficiency of miR-1245b-5p was validated by qRTPCR. (K and $\mathbf{L}$ ) CASK mRNA expression and protein levels were determined by RT-qPCR and Western blot in sh-NC, sh-AATBC and sh-AATBC+miR-1245b-5p inhibitors groups. (M) The knockdown competence of CASK was validated by qRT-PCR. (N-Q) The influence of biological function including colony formation $(\mathbf{N})$, apoptosis $(\mathbf{O})$, migration $(\mathbf{P})$ and invasion $(\mathbf{Q})$ were determined in $\mathrm{PCa}$ cells with the silence of CASKC. ${ }^{* * P}<0.0$ I.

\section{Mir-1245b-5p/CASK Axis Mediated Oncogenic Roles of AATBC in PCa}

To further illustrate the effect of AATBC/miR-140-5p/

IGF1R axis in PCa progression, we proposed to perform rescued experiments. The overexpressed CASK plasmids (oe-CASK) were constructed and the efficacy of CASK overexpression was judged via qRT-PCR (Figure 4A). As shown in Figure $4 \mathrm{~B}$ and $\mathrm{C}$, the abrogated cell colony formation produced through AATBC depletion was reversed via the reduced miR-1245b-5p or expanded CASK. What is more, flow cytometry analysis illustrated that the increased cell apoptosis induced via AATBC diminution was overturned by the miR-1245b-5p down- regulation or CASK amplification (Figure 4D). Finally, transwell assay manifested that the miR-1245b-5p knockdown or CASK overexpression could neutralize the restrained capacity for migration and invasion triggered via AATBC knockdown (Figure 4E and F). Collectively, AATBC promoted the growth and migration of $\mathrm{PCa}$ by the mediation of the miR-1245b-5p/CASK axis.

\section{Discussion}

The roles of IncRNAs in the development, progression, diagnosis and prognosis of $\mathrm{PCa}$ have been increasingly reported. For example, lncRNA NAP1L6 is upregulated and associated with the biochemical recurrence-free 
A

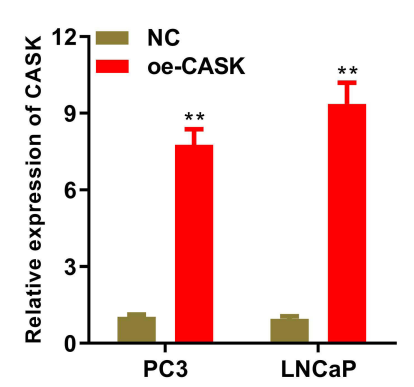

B

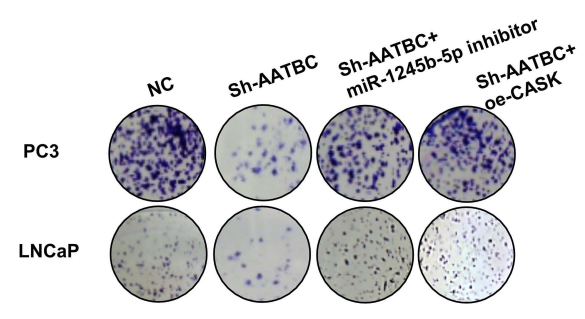

C

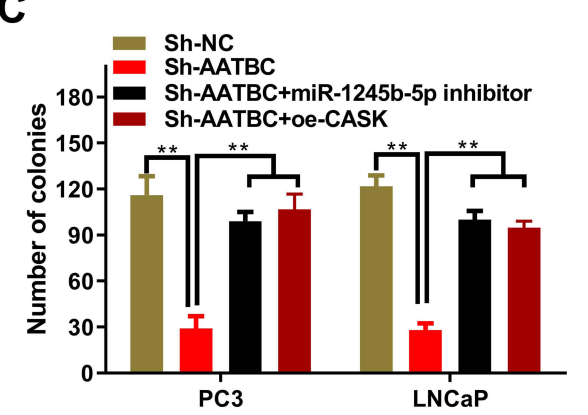

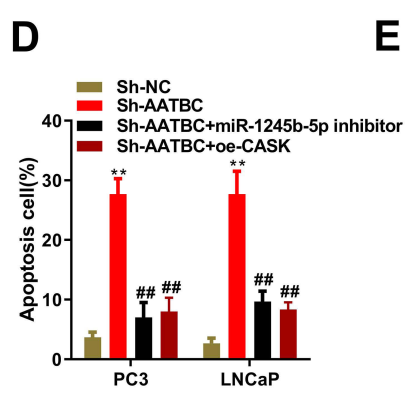

$\mathbf{F}$
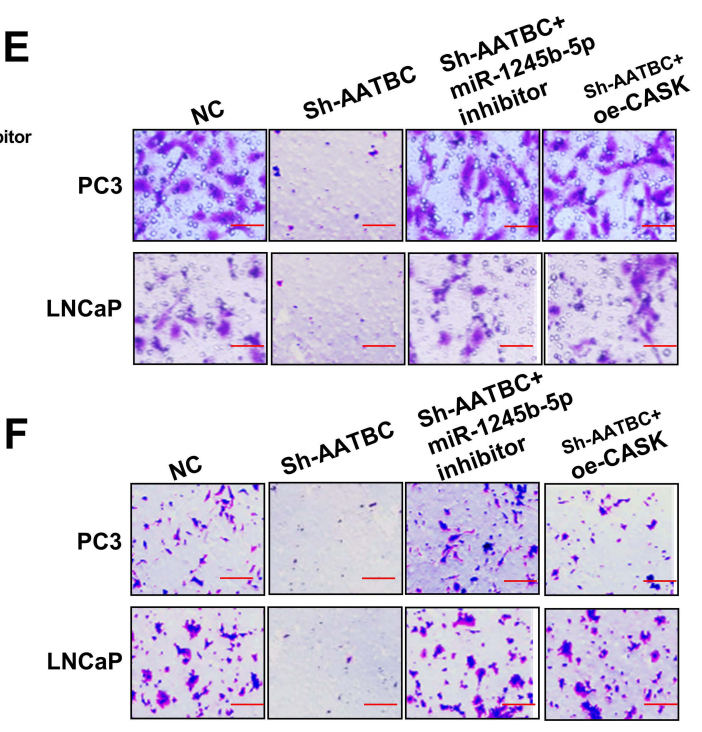
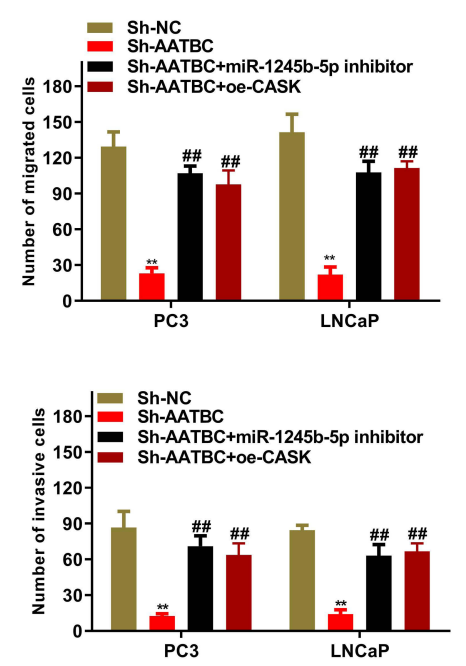

Figure 4 AATBC advanced PCa cell progression by the regulation of miR-1245b-5p/CASK axis. (A) The efficiency of CASK overexpression was manifested by RT-qPCR. (B and C) Colony formation was carried out to weigh cell proliferation competence in PCa cells of AATBC depletion plus miR-1245b-5p downregulation or CASK overexpression. (D) The apoptosis in PCa cells of AATBC depletion plus miR-1245b-5p downregulation or CASK overexpression was determined by the analysis of Annexin V-FITC/PI apoptosis detection kit. (E and F) The migratory and invasive ability of PCa cells of AATBC depletion plus miR-I245b-5p downregulation or CASK overexpression

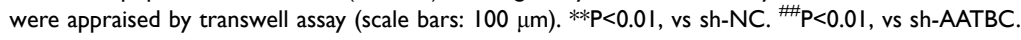

survival, and enhances the expression of INHBA to augment the growth in PCa. ${ }^{18}$ LncRNA SNHG12 predicts the poor prognosis of $\mathrm{PCa}$ and exerts its oncogenic effects via sponging miR-133b. ${ }^{19}$ Lnc-LBCS is low-regulated and associated with good prognosis in castration resistance $\mathrm{PCa}$ and can interact with hnRNPK to inhibit the activation of AR signal pathway. ${ }^{20}$ AATBC as a potential prognostic biomarker or therapeutic target of nasopharyngeal carcinoma or bladder cancer has been reported in the previous studies. ${ }^{16,17}$ In our present, we were the first to probe into the role of AATBC in PCa cells. All data suggested that AATBC was overexpressed, and exerted as a cancer-promoting gene in PCa.

MiRNAs as molecular sponges of lncRNAs have been investigated in voluminous studies. As an example, long noncoding RNA LINC00941 can competitively bind with miR-335-5p, leading to the upregulation of ROCK1 and the activation of LIMK1/Cofilin-1 signaling. ${ }^{21}$ LncRNA
ZFPM2-AS1 sponges miR-139 to regulate the expression of GDF10, and further stimulates hepatocellular carcinoma progression. ${ }^{22}$ In addition, miRNAs seem to be novel diagnostic/therapeutic instruments as they could play a role as a biomarker in the diagnosis and may also represent possible targets for new therapies in order to reduce the mortality of $\mathrm{PCa}^{23-25}$ In this present, miR$1245 \mathrm{~b}-5 \mathrm{p}$ was confirmed as the target of AATBC by bioinformatic analysis and subsequent experiment validation. In addition, we also observed the cancer-suppressive role of miR-1245b-5p by gain-of-function assay in $\mathrm{PCa}$ cells. In the previous study, miR-1245b-5p was observed to be associated with herpes simplex virus (HSV) 2 infection, ${ }^{26}$ but the expression and role of miR-1245b-5p in cancer was unknown. Therefore, we were the first study to report the functional role of miR-1245b-5p in tumors, and observed that miR-1245b-5p played a cancersuppressing role in $\mathrm{PCa}$. 
Subsequently, the downstream target of miR-1245b-5p was explored by bioinformatic tools, and CASK was validated as the only target in PCa cells. Calcium/calmodulindependent serine protein kinase (CASK), a member of membrane-associated guanylate kinase (MAGUK) superfamily, is implicated in regulating cell proliferation, cytoskeletal remodeling, and cell metastasis. In the previous studies of CASK in esophageal carcinoma and pancreatic adenocarcinoma, CASK was overexpressed and associated with the tumorigenesis of cancer. ${ }^{27,28}$ Likewise, we observed that CASK was overexpressed in PCa cells, and loss-of-function assay revealed that CASK played an oncogenic role in the progression of PCa. Moreover, rescue assay depicted that miR-1245b-5p/CASK axis mediated the oncogenic roles of AATBC in PCa. MiR1245b-5p overexpression or CASK silencing could restore the regulatory effect of IncRNA AATBC silence on cell proliferation, apoptosis, migration and invasion of $\mathrm{PCa}$.

But this study had some limits. We only detected the expression of miR-245b-5p and CASK in cell lines, whereas the expression in tumor tissues was not detected. In addition, the vivo study to prove the effect of miR$1245 b-5 p / C A S K$ axis would be explored in our future study.

All in all, our present demonstrated that AATBC stimulated $\mathrm{PCa}$ cell progression via regulating the miR$1245 \mathrm{~b}-5 \mathrm{p} / \mathrm{CASK}$ axis, which connoted that AATBC might be a novel and promising therapeutic target for PCa therapy.

\section{Data Sharing Statement}

The data that support the findings of this study are available from the corresponding author upon reasonable request.

\section{Funding}

This study received the financial support from the Special Diseases Program of Pudong New Area Health System (No. PWZzb2017-06).

\section{Disclosure}

All authors declare that they have no conflicts of interest.

\section{References}

1. Rawla P. Epidemiology of prostate cancer. World J Oncol. 2019;10 (2):63-89. doi:10.14740/wjon 1191
2. Nevedomskaya E, Baumgart SJ, Haendler B. Recent advances in prostate cancer treatment and drug discovery. Int $J$ Mol Sci. 2018;19(5). doi:10.3390/ijms19051359

3. Sumanasuriya S, De Bono J. Treatment of advanced prostate cancer-a review of current therapies and future promise. Cold Spring Harb Perspect Med. 2018;8(6). doi:10.1101/cshperspect. a030635

4. Chan JJ, Tay Y. Noncoding RNA:RNA regulatory networks in cancer. Int J Mol Sci. 2018;19(5). doi:10.3390/ijms19051310

5. Anastasiadou E, Jacob LS, Slack FJ. Non-coding RNA networks in cancer. Nat Rev Cancer. 2018;18(1):5-18. doi:10.1038/ nrc.2017.99

6. Chi Y, Wang D, Wang J, Yu W, Yang J. Long non-coding RNA in the pathogenesis of cancers. Cells. 2019;8(9). doi:10.3390/cells8091015

7. Kumar MM, Goyal R. LncRNA as a therapeutic target for angiogenesis. Curr Top Med Chem. 2017;17(15):1750-1757. doi:10.2174/1568026617666161116144744

8. Xiao ZD, Zhuang L, Gan B. Long non-coding RNAs in cancer metabolism. Bioessays. 2016;38(10):991-996. doi:10.1002/ bies. 201600110

9. Bhan A, Soleimani M, Mandal SS. Long noncoding RNA and cancer: a new paradigm. Cancer Res. 2017;77(15):3965-3981. doi:10.1158/ 0008-5472.CAN-16-2634

10. Zhao J, Du P, Cui P, et al. LncRNA PVT1 promotes angiogenesis via activating the STAT3/VEGFA axis in gastric cancer. Oncogene. 2018;37(30):4094-4109. doi:10.1038/s41388-018-0250-z

11. Kong R, Zhang EB, Yin DD, et al. Long noncoding RNA PVT1 indicates a poor prognosis of gastric cancer and promotes cell proliferation through epigenetically regulating p15 and p16. Mol Cancer. 2015;14:82. doi:10.1186/s12943-015-0355-8

12. Wang Z, Cheng Y, Zhu Y, et al. Long non-coding RNA FOXD1-AS1 promotes the progression and glycolysis of nasopharyngeal carcinoma by sustaining FOXD1 expression. Am J Cancer Res. 2020;10 (11):3686-3704

13. Gao YF, Liu JY, Mao XY, et al. LncRNA FOXD1-AS1 acts as a potential oncogenic biomarker in glioma. CNS Neurosci Ther. 2020;26(1):66-75. doi:10.1111/cns.13152

14. Xie J, Zhong Y, Chen R, et al. Serum long non-coding RNA LINC00887 as a potential biomarker for diagnosis of renal cell carcinoma. FEBS Open Bio. 2020;10(9):1802-1809. doi:10.1002/ 2211-5463.12930

15. Li T, Liu N, Gao Y, et al. Long noncoding RNA HOTAIR regulates the invasion and metastasis of prostate cancer by targeting hepaCAM. Br J Cancer. 2020.

16. Zhao F, Lin T, He W, et al. Knockdown of a novel lincRNA AATBC suppresses proliferation and induces apoptosis in bladder cancer. Oncotarget. 2015;6(2):1064-1078. doi:10.18632/oncotarget.2833

17. Tang T, Yang L, Cao Y, et al. LncRNA AATBC regulates Pinin to promote metastasis in nasopharyngeal carcinoma. Mol Oncol. 2020;14(9):2251-2270. doi:10.1002/1878-0261.12703

18. Zheng Y, Gao Y, Li X, et al. Long non-coding RNA NAP1L6 promotes tumor progression and predicts poor prognosis in prostate cancer by targeting Inhibin-beta A. Onco Targets Ther. 2018;11:4965-4977. doi:10.2147/OTT.S163680

19. Cheng G, Song Z, Liu Y, et al. Long noncoding RNA SNHG12 indicates the prognosis of prostate cancer and accelerates tumorigenesis via sponging miR-133b. J Cell Physiol. 2020;235(2):1235-1246. doi:10.1002/jcp.29039

20. Gu P, Chen X, Xie R, et al. A novel AR translational regulator IncRNA LBCS inhibits castration resistance of prostate cancer. Mol Cancer. 2019;18(1):109. doi:10.1186/s12943-019-1037-8

21. Wang $\mathrm{J}, \mathrm{He} \mathrm{Z}, \mathrm{Xu} \mathrm{J}$, Chen $\mathrm{P}$, Jiang J. Long noncoding RNA LINC00941 promotes pancreatic cancer progression by competitively binding miR-335-5p to regulate ROCK1-mediated LIMK1/Cofilin-1 signaling. Cell Death Dis. 2021;12(1):36. doi:10.1038/s41419-02003316-w 
22. He H, Wang Y, Ye P, et al. Long noncoding RNA ZFPM2-AS1 acts as a miRNA sponge and promotes cell invasion through regulation of miR-139/GDF10 in hepatocellular carcinoma. $J$ Exp Clin Cancer Res. 2020;39(1):159. doi:10.1186/s13046-020-01664-1

23. Cochetti G, Poli G, Guelfi G, Boni A, Egidi MG, Mearini E. Different levels of serum microRNAs in prostate cancer and benign prostatic hyperplasia: evaluation of potential diagnostic and prognostic role. Onco Targets Ther. 2016;9:7545-7553. doi:10.2147/OTT. S119027

24. Cochetti G, Rossi de Vermandois JA, Maula V, et al. Role of miRNAs in prostate cancer: do we really know everything? Urol Oncol. 2020;38(7):623-635. doi:10.1016/j.urolonc.2020.03.007

25. Guelfi G, Cochetti G, Stefanetti V, et al. Next Generation Sequencing of urine exfoliated cells: an approach of prostate cancer microRNAs research. Sci Rep. 2018;8(1):7111. doi:10.1038/s41598-018-24236-y
26. Kuang L, Deng Y, Liu X, Zou Z, Mi L. Differential expression of mRNA and miRNA in guinea pigs following infection with HSV2v. Exp Ther Med. 2017;14(3):2577-2583. doi:10.3892/etm.2017.4815

27. Wang Q, Lu J, Yang C, et al. CASK and its target gene Reelin were co-upregulated in human esophageal carcinoma. Cancer Lett. 2002;179(1):71-77. doi:10.1016/S0304-3835(01)00846-1

28. Meng M, Liu S, Wang C, Gu X, Linghu E, Xue X. Mass spectrum analysis of membrane proteins reveals that CASK, CD36 and EPB42 are differentially expressed in pancreatic adenocarcinoma. Oncol Lett. 2020;20(6):376. doi:10.3892/ol.2020.12239

\section{Publish your work in this journal}

Cancer Management and Research is an international, peer-reviewed open access journal focusing on cancer research and the optimal use of preventative and integrated treatment interventions to achieve improved outcomes, enhanced survival and quality of life for the cancer patient.
The manuscript management system is completely online and includes a very quick and fair peer-review system, which is all easy to use. Visit http://www.dovepress.com/testimonials.php to read real quotes from published authors. 